\title{
THE READOUT SYSTEM FOR THE MULTIWIRE PROPORTIONAL CHAMBERS OF THE CERN SPS SECONDARY BEAMS
}

\author{
L. PREGERNIG and M. RABANY \\ CERN, Geneva, Switzerland
}

\begin{abstract}
To provide CERN-SPS experiments with accurate momentum measurement of incoming beam particles associated to their events, we had to take into consideration the high fluxes in the secondary beams $\left(<10^{7} \mathrm{pps}\right)$ and the multi-user access to the spectrometer data.

The very fast readout system described here, permitting decisions at several levels, fulfills these requirements.
\end{abstract}

\section{Introduction}

In most of the SPS secondary beams the momentum determination of individual particles is done by means of focussing spectrometers using four wire chambers as detectors ${ }^{1}$ ). The specifications required are $1 \mathrm{~mm}$ wire spacing and up to $10^{7}$ particles per second. The chambers of one spectrometer are spread over more than a hundred meters. Consequently, memorisation cannot be effected locally and all signals have to be sent to one central point (see also ref. 2).

Apart from the special attention brought to the electronics associated to the very weak signals which came out of the multiwire proportional chamber at high rate, much care has been taken on the readout system, with in mind the following considerations.

1) Any experimenter of one beam should see the spectrometer as if it was his own;

2) the system should be as much as possible independent of the number of chambers and of the number of wires per chamber, these two numbers being optimised for each spectrometer;

3) in case of trouble, the system should permit fast diagnostics.

\section{Design of the read-out system}

\subsection{MUlTIUSER ACCESS}

\subsubsection{Dead time}

Different users may share one common equipment if none of them is severely disturbed by the others. For each request of any user, the equipment is dead for the others during a time $t$. If $m$ is the number of users, each of them sending $N$ requests per second, the efficiency of the system will be:

$\eta=\frac{1}{1+N(m-1) t}$.

As $\eta$ must be $\simeq 1, \eta \simeq 1-N(m-1) t$, and

$t \simeq \frac{1-\eta}{N(m-1)}$

The efficiency loss has to be very small to be acceptable, say less than $1 \%$, let us take $1-\eta=0.002$ with three users and a maximum of $10^{3}$ requests/s we get

$t=\frac{0.002}{10^{3} \times 2}=1 \mu \mathrm{s}$.

Thus $1 \mu \mathrm{s}$ after one user's request, the system must be free again. As we have nominally one data per chamber and four chambers, the read-out time should be of the order of $150 \mathrm{~ns} /$ data, taking into account multiplicity and some overhead.

\subsubsection{Independency}

Each user must have the possibility, without disturbing the others:

1) to vary his strobe width;

2) to delay his strobe;

3) to clear rapidly, if the event is rejected at another level of decision;

4) to use the signals coming out of the chambers as part of the decision.

This means the existence of an arbitration module to which all users have to send their requests. This box has then to take care of all the mastership problems and to control the timing of the read-out. Furthermore, as the strobe signal must pass through without being influenced on its time 


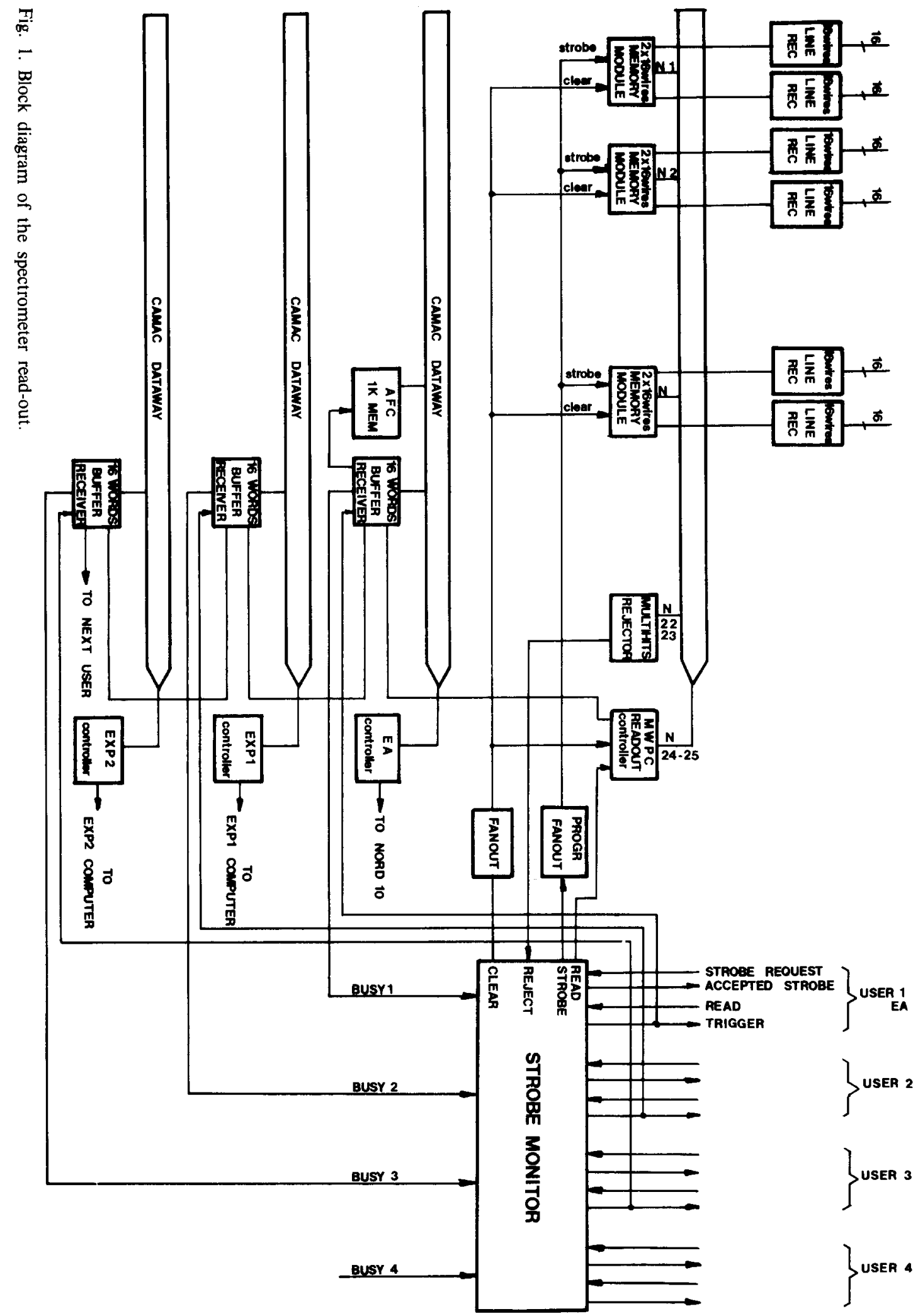




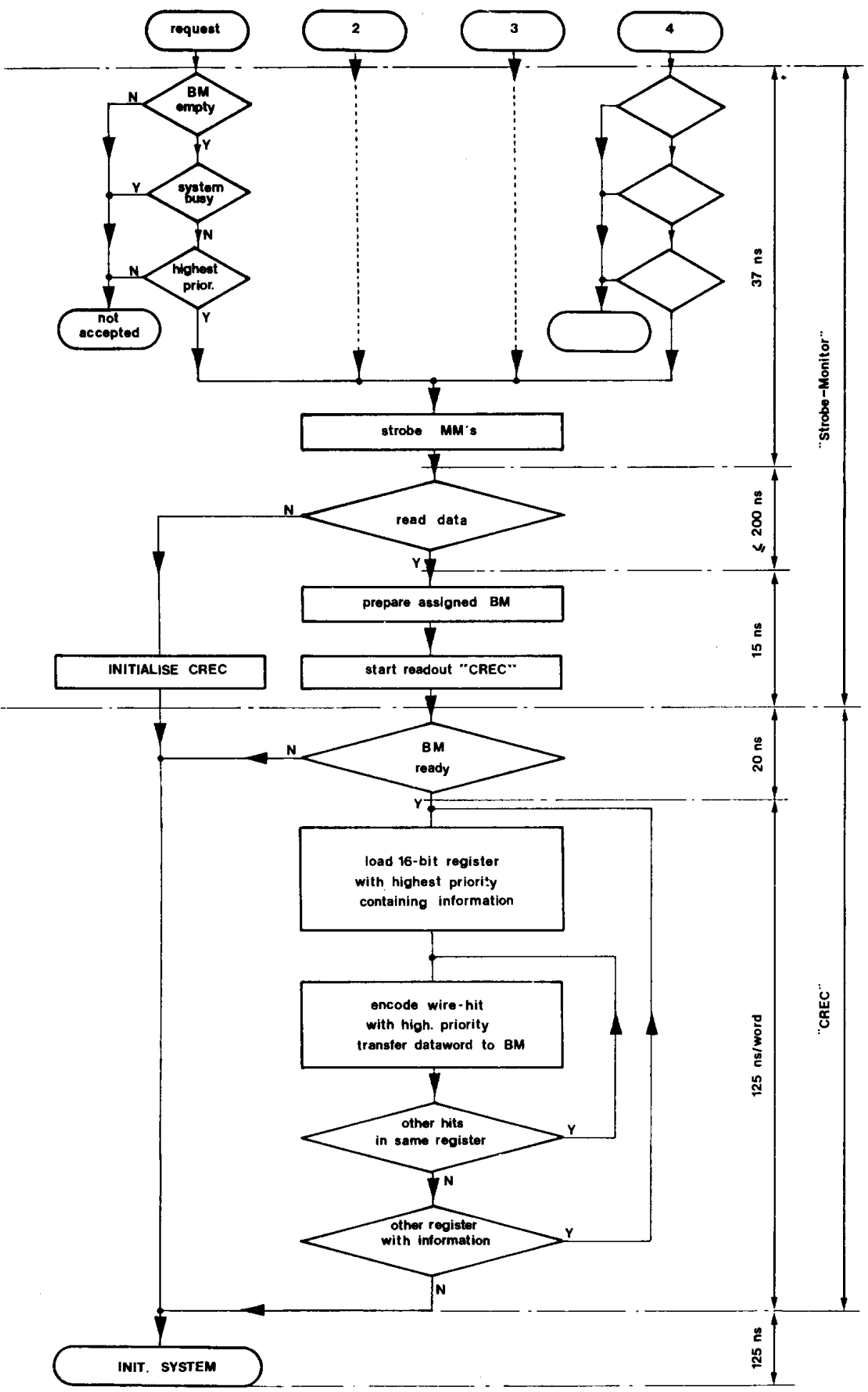

Fig. 2. Flow-chart of the read-out sequence. 
parameters, special attention has to be paid in order to avoid transit drifts.

\subsection{MOdULARITY}

One typical beam spectrometer is composed of four chambers having in total 320 useful wires. But in practice this number varies from 160 to 640 according to beam sizes and number of chambers. The system must then be very flexible to fit these various arrangements.

\subsection{FAST DiagnOSTIC}

The momentum measurement system is a permanent facility offered to users. As such, in order to find rapidly eventual faults, diagnostics have to be performed with the computer assitance. This means that all important parameters have to be controlled by the computer.

\section{Description of the read-out system}

\subsection{System configuration}

Generally for one spectrometer 4 multiwire proportional chamber-planes, with 64 or 96 wires per plane, are used. Due to the high beam fluxes a read-out system with one channel for each wire has been chosen.

Because of the long distances (several hundred meters between detectors and electronics-hut) preamplifiers and discriminators are located at the detector-side.

As shown in fig. 1 the "Memory-Modules" and the crate-controller "CREC" perform the proper read-out function, whereas "Strobe-Monitor" and "Buffer Memories" are considered as multiuseraccess elements.

To access the spectrometer data the physicist sends a request to the "Strobe-Monitor". This request, after it has been accepted, is used to strobe the wire-information into the "Memory-Modules", each of them containing two 16-bit registers.

One crate, a standard CAMAC-crate, contains up to 23 Memory-Modules (MM) and one cratecontroller "CREC" (Chamber-Read-out-Encoder-Controller). Communication between the MMs and the CREC takes place on the TWISTEDPAIR dataway of the crate.

The CREC will allocate a binary number based on channel number in register, register number in module, module number in crate - to each wire which has been hit.
The action of the CREC is initiated from the Strobe-Monitor. This unit which maintains the requested strobe-width and also settles the priority of coinciding requests, allows on a second level (at latest $200 \mathrm{~ns}$ after the strobe) to decide whether to read the data or to clear the MMs. A "multihit" logic, based on the information already stored in the MMs, can be introduced in this decision to reject events characterised by too many wires hit in the same plane.

Upon a "Read"-decision the CREC extracts the data in a LAM-priority-encoding mode at a rate of $125 \mathrm{~ns} / 16$ bits word (= wire hit) and transmits the encoded wire numbers into the "Buffer-Memory" $(\mathrm{BM})$ assigned to the requesting user and "prewarned" by the strobe-monitor.

This fast Buffer-Memory ( $32 \times 16$ bits words, access time about $100 \mathrm{~ns}$ ) is normally situated in the user's CAMAC-crate where it can be read directly through a standard CAMAC-controller. But it may also be operated as a serial transmitter or receiver, when the distance between the CREC and the user's CAMAC exceeds $15-20 \mathrm{~m}$. The complete read-out sequence is shown in the flow-chart fig. 2.

The system deadtime depending on the number of valid data words and the decision time (individual for each user) can be calculated as:

Dead time $=$ decision time $+300 \mathrm{~ns}+(n-1) \times$ $\times 125 \mathrm{~ns}$, with $n$ the number of datawords.

\subsection{Choice of Electronic-STANDARD}

The system has been designed in SchottkyTTL-logic which makes the MMs CAMAC compatible (with jumper-option for fast read-out). The inputs of the MMs are differential ECL-inputs terminated with $100 \Omega$.

\subsection{Description of the modules \\ 3.3.1. Strobe-Monitor (fig. 3)}

This single-width NIM-unit controls the multiuser-access to the spectrometer-information. It has to prevent from multiple strobing, to maintain the width of the requested strobe-signal, which the user may vary for different reasons, and to prepare for the data to be sent to the requesting user exclusively. Input sections for four users are provided. To access the spectrometer the user sends a request-signal with the width corresponding to the desired strobe width for the MMs, which will be accepted, if the system is not busy and if - in the case of several coinciding requests - it has the 

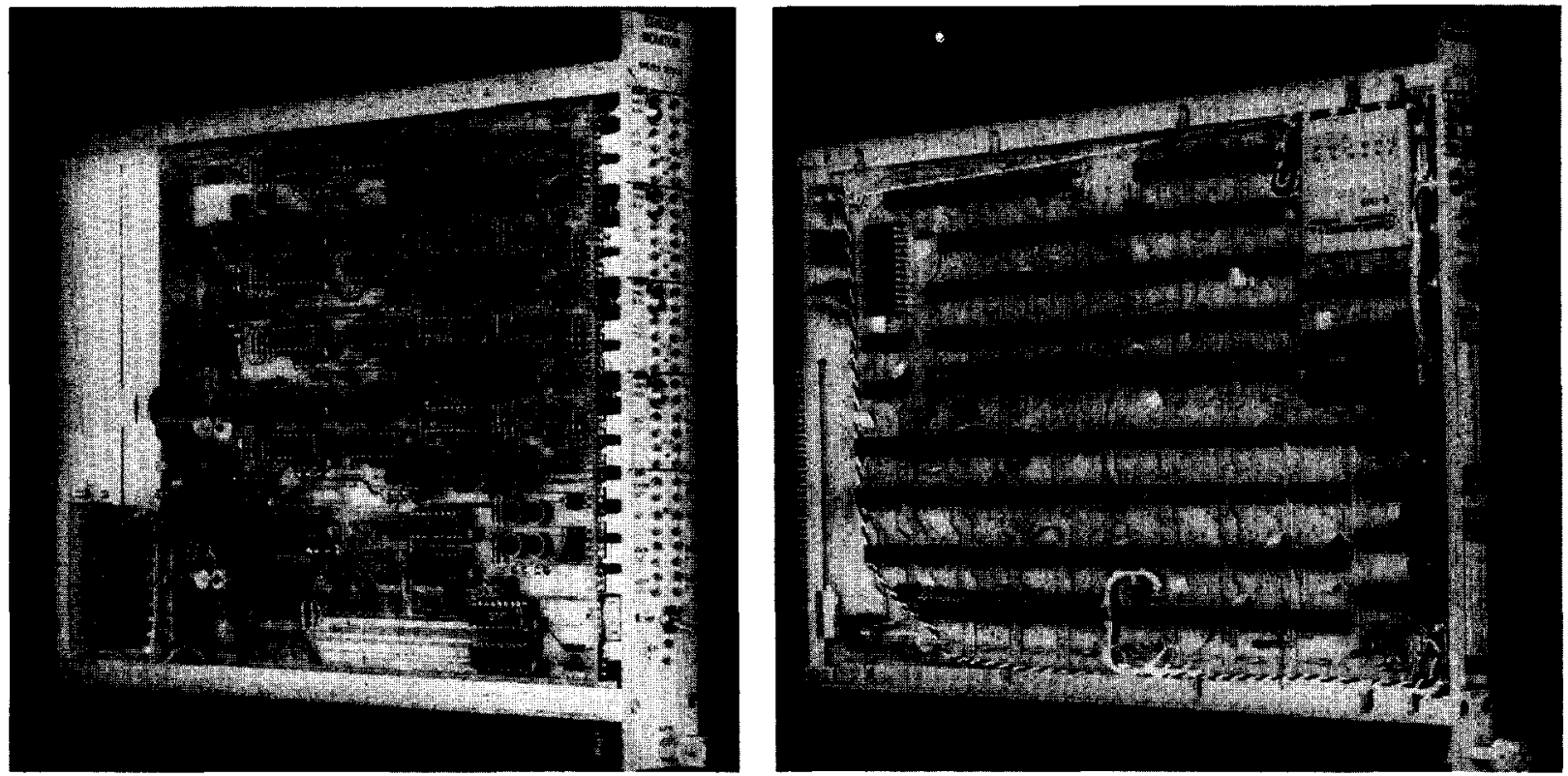

Fig. 3. Buffer-Memory and Strobe-Monitor.
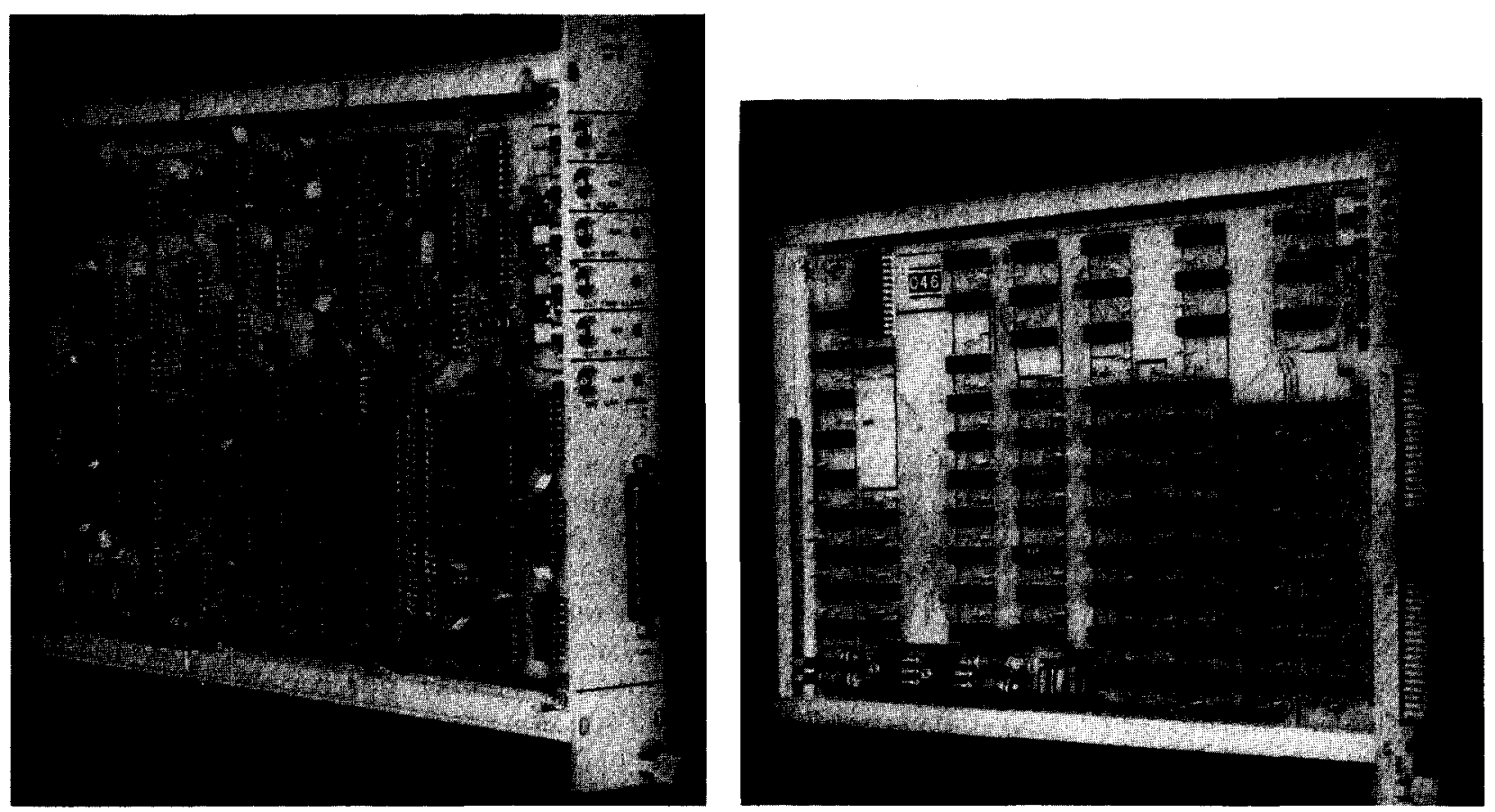

Fig. 4. Memory-module and CREC.

highest priority. Additionally the user may decide by sending or not a "Read"-signal (at a time $\leq 200 \mathrm{~ns}$ after Strobe) whether to read the data or to reset the system.

During the system-dead time no strobe-request will be accepted, a "read" causes the input section of the requesting user to remain locked until the corresponding Buffer-Memory has been emptied or cleared.

\subsubsection{Memory-module (fig. 4)}

This single-width CAMAC compatible unit con- 


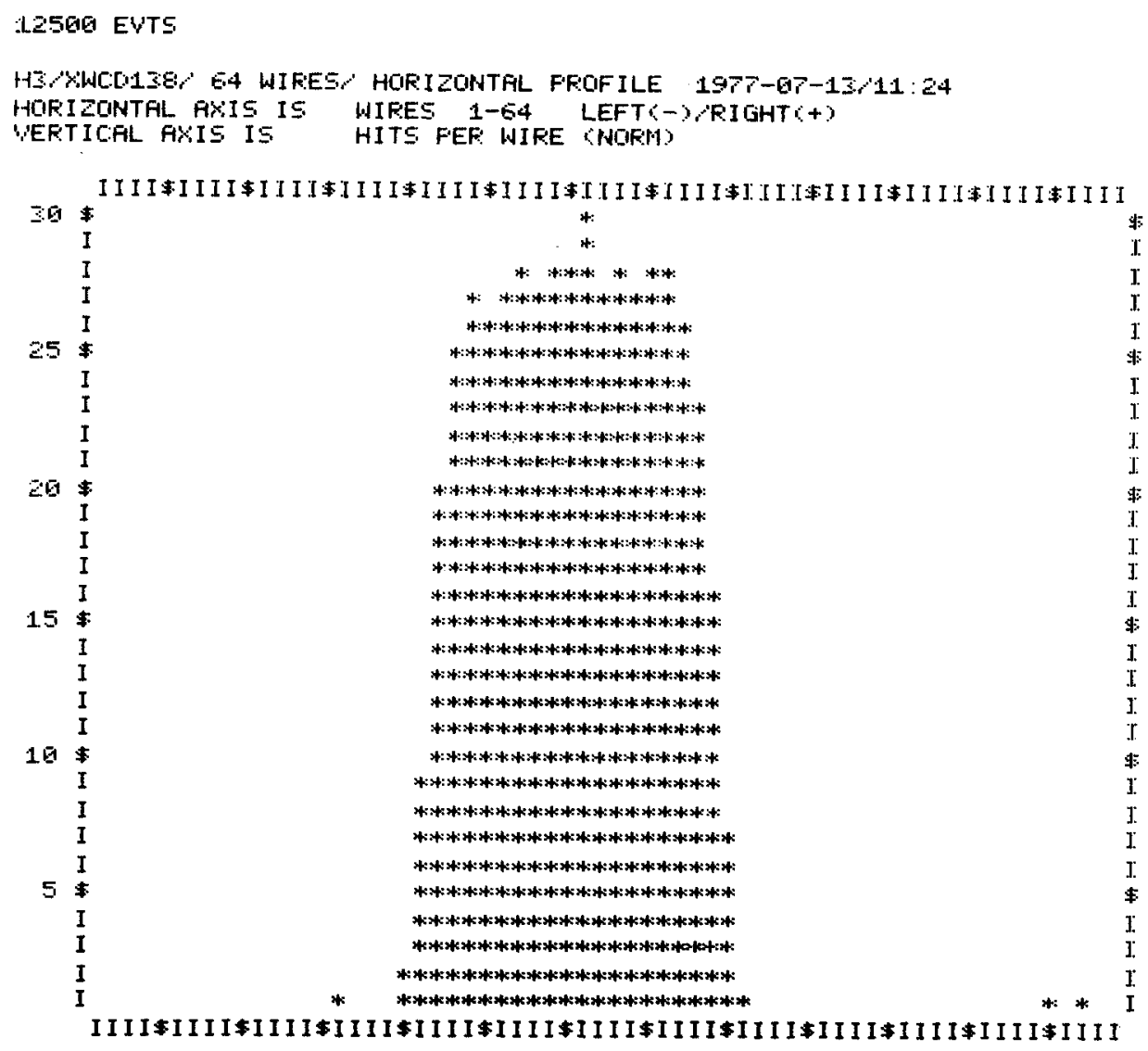

MOMENTUM OF DISTR. $=-2$. $90 \mathrm{MM}$, RMS $=6.71 \mathrm{MM}$

Fig. 5. Beam profile at one detector plane.

tains two 16-bit registers words as intermediate storage device for the wire-information. The wiresignals, differentially driven ECL-signals, are translated to TTL-levels and will set a register-bit when the leading edge of the wire signal and the strobe coincide (strobe-width $\geq 10 \mathrm{~ns}$ ). The two 16 registers act as independent sources for the module-LAM, when their content is different from 0 . A multihit-logic, counting adjacent wirehits as one, calculates within $90 \mathrm{~ns}$ after the strobe the number of hits - CARRY IN and OUT of this information is available for daisy chaining.

\subsubsection{CREC (fig. 4)}

The function of the CREC is to read out information that is present in a crate of MMs, to code the information and to send it for storage to the Buffer-Memories. The module is two units wide. The command to read the MMs, given by the Strobe-Monitor, initiates the following sequence.
After a delay of $100 \mathrm{~ns}$ to allow for the MMLAMs to be settled, the controller loads - performing a "read and clear" dataway-cycle - the bit-pattern of the register with the highest priority (depends on the position within the crate). This data pattern is encoded by priority encoders to present the least significant data bit in binary form. This binary number (4 bits) combined with the encoded register number (6 bits), giving a unique solution for a wire within a crate, is transferred to the Buffer-Memory. Immediately afterwards this active bit has been erased, so that the next active bit may be encoded or if there is no more active bit, the next active register may be loaded. The data transfer rate to the Buffer-Memory is $125 \mathrm{~ns} / 16$-bit word.

\section{Dataformat:}

First word flag $\quad-1$ bit.

Last word flag $\quad-1$ bit. 


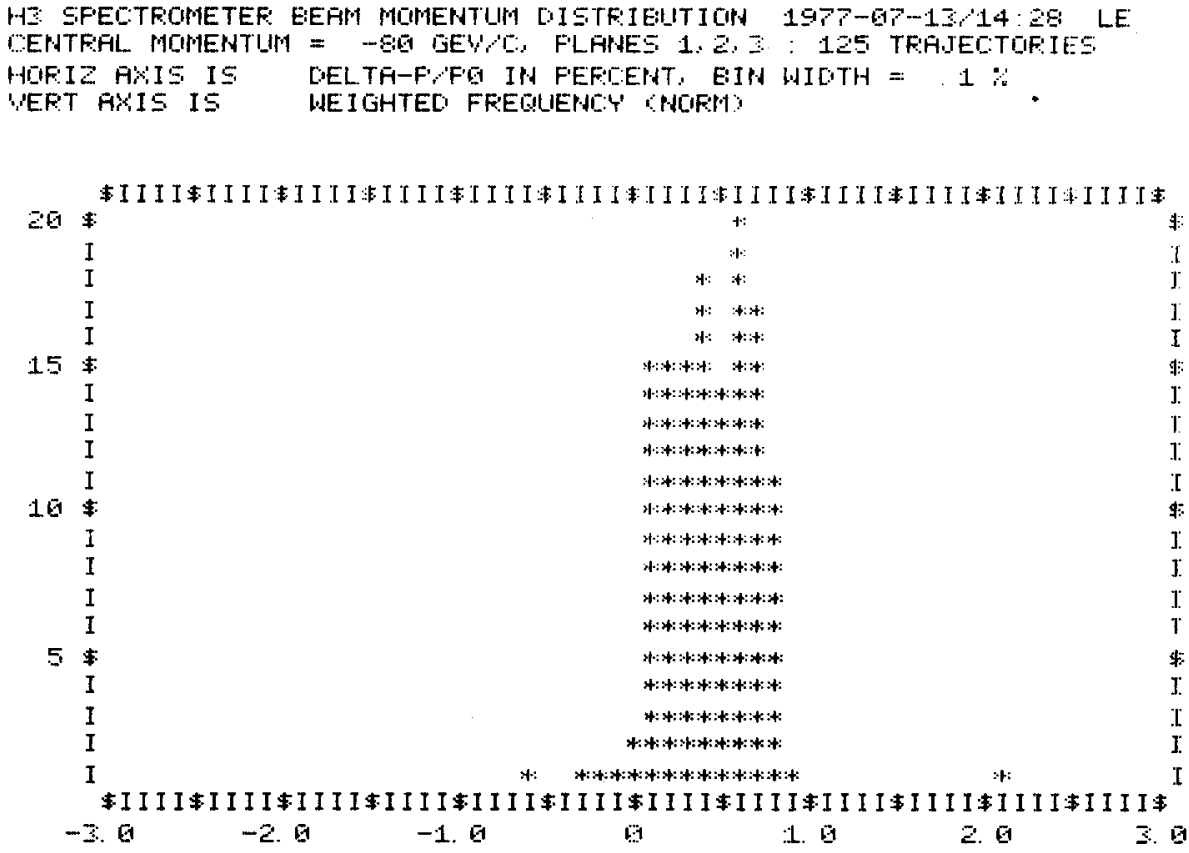

WEIGHTED MEFN V'FLUE OF DELTF-F,FE $=.39 \%$, DISTE. FIS $=.30$ Fig. 6. $\Delta p / p$ distribution.

$\begin{array}{ll}\text { No hit flag } & -1 \text { bit. } \\ \text { (Unused) } & -3 \text { bits. } \\ \text { Slot no. } & -5 \text { bits. } \\ \text { Reg. no. } & -1 \text { bit. } \\ \text { Bit position within register } & -4 \text { bits. }\end{array}$

Event-blocks are marked by the First word and Last word-flag on in the corresponding data words.

\subsubsection{Buffer-Memory (fig. 3)}

This single-width CAMAC module has been designed as a fast 32 words-16 bits first-in-last-out buffer. It can be operated in one of the following modes:

- CAMAC-Receiver - Serial Transmitter. Data written into the memory by CAMAC-write commands are transmitted serially (asynchronous, $5 \mathrm{M}$-bits, optocouplers) to another Buffer-Memory, which works as

- Serial-Receiver - CAMAC-Transmitter. Data received from a serial transmitter and written into the memory are read by CAMAC-Read Commands.

- Parallel-Receiver - CAMAC-Transmitter. Parallel data written into the memory via the CannonFront-Panel Connector are read by CAMACRead Commands.

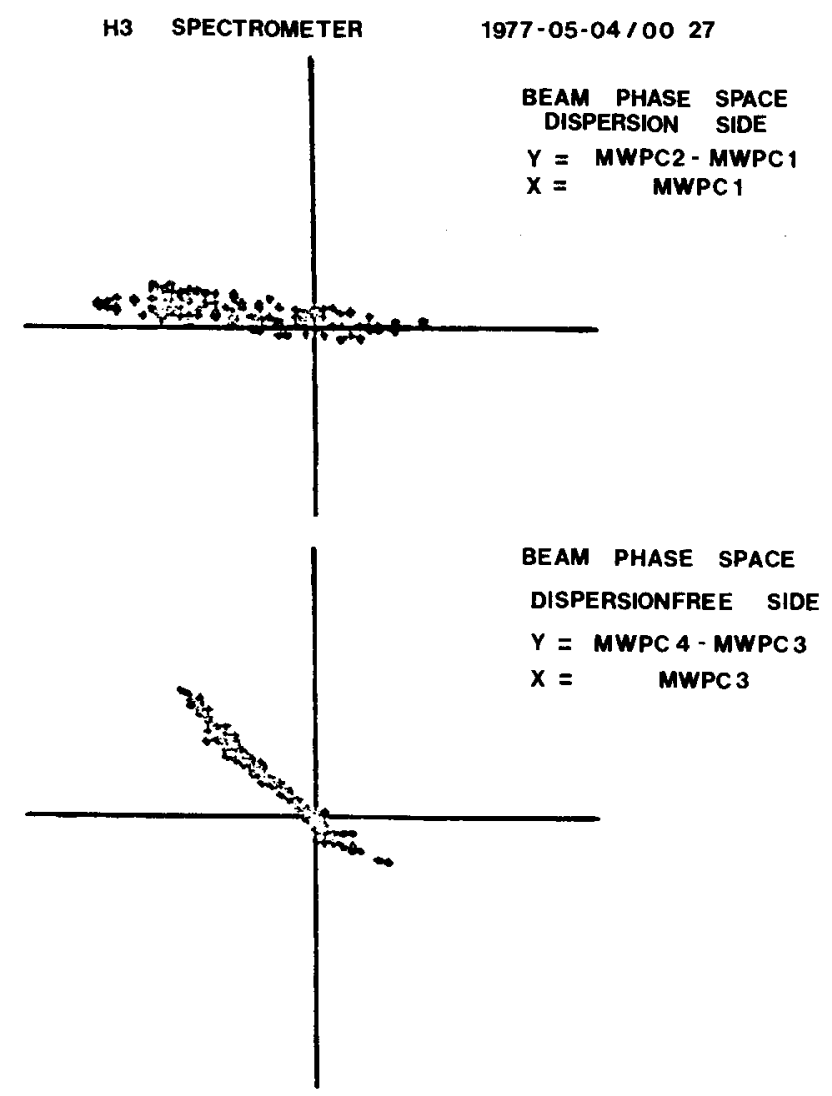

Fig. 7. Phase-space plot. 
- Parallel-Receiver - Serial-Transmitter. Parallel data written into the memory via the CannonConnector on the front panel are transmitted serially to another Buffer-Memory. Serial transmission starts automatically on reception of the Last-word flag.

\section{Conclusion}

Five spectrometers are actually operational on SPS secondary beam lines in the West Experimental Area. Several others are prepared for installation in the North Experimental Area.
The systems seem to work satisfactorily. Maintenance is quite easy through the computer capable of displaying various histograms and tables which permit to check rapidly the behaviour of the systems (figs. 5, 6, 7).

\section{References}

l) J. V. Allaby and C. Bovet, CERN internal report, CERN/Lab. II/EA/74-5.

2) G. Dubois, A. Placci, L. Pregernig, M. Rabany, B. Skaali and G. Vismara, Proc. Symp. on Nuclear science, San Francisco (Oct. 19-21, 1977). 